TM-1520

[SSC-N-506]

\title{
Studies of One-Campus and Two-Level Configurations for SSC
}

\author{
L. C. Teng \\ Fermi National Accelerator Laboratory \\ P.O. Box 500, Batavia, Illinois 60510
}

April 29, 1988 
SSC-N-506

TU -1520

$4 / 29 / 88$

Studies of One-Campus and Two-Level Configurations for SSC

L. C. Teng

In this report we investigate in some detail the geometry and the cost of two modifications in the configuration of the SSC.

1) One-campus configuration--the possibility and the advantages of this configuration were first pointed out by L. Lederman ${ }^{1}$ and later further elaborated by L. Lederman and $L$. Teng ${ }^{2}$. Assuming, as in the Conceptual Design, that the fully developed and exploited SSC has 6 interaction regions (IR's) equipped with detectors and that for about half of the time a detector needs to be serviced and/or modified, one should then have a two-branch bypass with 3 IR's on each branch. And to minimize the campus length one should inject into 2 utility straight sections located on the trunk line just beyond the ends of the bypass, denoted as Case $\mathrm{C}$ in Ref. 2. The odd number of vertical crossings in each branch brings opposite rings to the top-side for identical injection geometry from above (or below) and yields good overall symmetry between the two rings. This implies, of course, that there must be another crossing (presumably without collision) in the long long straight section diametrically opposite from the Campus.

2) Two-level configuration--with the one-campus configuration the circumference of the collider is clearly divided into two sectors with totally different functions. The Campus sector serves all the active functions such as injection, acceleration, beam abort, beam collision and the performance of physics experiments with massive detectors. The remaining sector and by far the greater part of the 
circumference is just a big beam transport and serves only the inert function of channelling the beam leaving the Campus back to the Campus. Because of the difference in function the optimal physical arrangements for the active "Campus" and the inert "Transport" sectors may be quite different. Specifically, the optimal elevation for these two sectors are different: the active Campus should be easily accessible and hence closer to the grade level, and the inert Transport should be deeper underground to minimize interference with the use and habitation of the ground above.

For the basic SSC we assume the following parameters ${ }^{3}$ (each ring):

Phase advance per normal cell $90^{\circ}$

Half cell length, $\ell$ $114.25 \mathrm{~m}$

No. of dipoles per half cell

6

Bend angle per dipole, $\theta$ $1.64 \mathrm{mrad}$

Length of Interaction Region (IR) or Utility Region (UR) $11 \ell$

Length of Curved Region (CR) between IR's $9 \ell$

Bend angle per $C R$

Length of Arc Region (AR)

Bend angle per AR

Total number of dipoles

Circumference
500 (82 mrad)

$295 \ell$

$1766 \theta$ (2896 mrad)

3832

$732 \ell(83631 \mathrm{~m})$

We also need an all purpose matching region (MR). Such an MR is shown in Fig. 1. The section is composed of four $90^{\circ}$ cells with 6 halfcells filled with bending dipoles. This section matches zero dispersion $\left(D=D^{\circ}=0\right)$ to zero dispersion and has unity transfer matrices in both planes independent of the length $\ell_{M}$ and the bend angle $\theta_{M}$ which can 
be adjusted to match the geometry. We shall identify a specific matching region by $\mathrm{MR}\left(\ell_{\mathrm{M}}, \theta_{\mathrm{M}}\right)$.

\section{One-Campus Configuration (One-Level)}

\section{A) Symmetric Bypass}

The principal function of the $\mathrm{CR}$ in the basic SSC lattice is to eliminate interference from muons produced in neighboring IR's. But as we shall see later (Appendix A) the $C R$ as specified is longer than necessary. For the symmetric bypass shown in Fig. 2 we choose to use MR $(6 \ell, 24 \theta)$ 's to connect IR's. The IR length is held fixed so that the beam combining geometry and the low- $\beta$ and the medium- $\beta$ quadrupole arrangements are unchanged. The same is true with the UR. The parameters of the MR are chosen so that the end Interaction Points (IP's) are separated by a convenient distance. With these choices the parameters of the symmetric bypass are (each ring):

Minimum length of Campus to include all IP's $=34 l=3.9 \mathrm{~km}$

Separation of the end IP's $=\mathrm{s}=76.4 \mathrm{~m}$

Increment of total tunnel length over the basic $\mathrm{SSC}=\Delta \mathrm{L}=123 \mathrm{l}=$ $14.1 \mathrm{~km}$

Increment of total bend angle over the basic SSC $=\Delta \theta=192 \theta=$ $315 \mathrm{mrad}$

RMS $\mu$-beam radius at neighboring IP $=r_{\mu}=4.6 \mathrm{~m}($ Beam $/ \mathrm{IP}$ separation $=38 \mathrm{~m}>8 \mathrm{r}_{\mu}$ )

B) Unipolar Bypass

In this case a larger bend angle between the end UR's and IR's helps to create the separation between the branches. Therefore we will keep the basic CR's with bend angle $\theta_{C}=50 \theta$ at these locations as 
shown in Fig. 3. The separation between the branches is then created by inserting the two staggered straight sections of lengths $\ell_{I}$ (inner branch) and $l_{O}$ (outer branch). The most straightforward way to match across $\ell_{1}$ and $\ell_{O}$ is to use the straight matching sections MR $\left(\ell_{1}, 0\right)$ and MR $\left(\ell_{0}, 0\right)$. We shall assume the same separation $\mathrm{s}=76.4 \mathrm{~m}$ for easy comparison with the symmetric case. This value is also close to the minimum required to accommodate the large detector halls on both IP's. The parameters so obtained are:

Outer branch straight length

$$
\ell_{0}=\frac{\cos \theta_{c}}{\sin \theta_{C}} \mathrm{~s}=930.0 \mathrm{~m}=8.14 l
$$

Inner branch straight length

$$
\ell_{I}=\frac{\cos { }^{2 \theta_{C}}}{\sin \theta_{C}} \mathrm{~s}=920.6 \mathrm{~m}=8.06 \ell
$$

Yinimum length of Campus to include all IP's

$$
=34 l=3.8 \mathrm{~km}
$$

Separation of the IP's $=s=76.4 \mathrm{~m}$

Increment of total tunnel length over the basic SSC $=\Delta \mathrm{L}=$ $139.6 \mathrm{l}=16.0 \mathrm{~km}$

Increment of total bend angle over the basic $\mathrm{SSC}=\Delta \theta=148 \theta$

$=243 \mathrm{mrad}$

RMS $\mu$-beam radius at:

Nearest neighboring IP $=r_{\mu 1}=4.6 \mathrm{~m}$

$\left(\right.$ Beam/IP separation $\left.=38 \mathrm{~m}>8 \mathrm{r}_{\mu 1}\right)$

Second neighboring IP $=r_{\mu 2}=7.2 \mathrm{~m}$

$$
\left(\text { Beam/IP separation }=76 \mathrm{~m}>10 \mathrm{r}_{\mu 2}\right)
$$


Two-Level Configuration

We assume that the active "Campus" of the ring, the section that includes the 6 IR's, is horizontal and at an elevation $d_{V}$ above that of the inert "Transport" which is also horizontal. We need a vertical dogleg at each end of the Campus to produce the elevation difference.

\section{A) Symmetric Bypass}

Referring to Fig. 4 we see that the end section ABCD is made into a symmetric dogleg in a plane tilted at an angle $\alpha$ from the horizontal. Such a dogleg will produce a vertical displacement. The matching region $\overparen{C E}$ should be on a plane tilted at angle $\pi-\alpha$ from horizontal. This reverses the vertical component of the field and leaves the horizontal component unchanged. Thus the horizontal projections of $\overparen{C D}$ and $\overparen{C E}$ will bend in opposite directions and the vertical projection of $A B C E$ will be identical to that of $A B C D$.

We will keep the axes of the quadrupoles untilted (no rotation about the beam axis) so that any coupling of beam optics in the two transverse planes is introduced only by the skewed bending of the dipoles. It should be easy to compensate for this weak coupling by the trim skew quadrupoles which are already included in the lattice.

The Tilted Regions $\overparen{A B}, \overparen{C D}$ and $\overparen{C E}$ are denoted by $T R$ and are formed by the very versatile all purpose matching regions with lattice given in Fig. 1. The length and bend angle will be denoted by $\ell_{T}$ and $\theta_{\mathrm{T}}$. The projected horizontal $\left(\theta_{\mathrm{H}}\right)$ and vertical $\left(\theta_{\mathrm{V}}\right)$ bend angles are given by (see Appendix B).

$$
\begin{aligned}
\tan \theta_{\mathrm{H}} & =\tan \theta_{\mathrm{T}} \cos a \\
\tan \theta_{\mathrm{V}} & =\tan \theta_{\mathrm{T}} \sin \alpha
\end{aligned}
$$


The total horizontal and vertical excursions $d_{H}$ and $d_{V}$ from $A$ to $D$ of the dogleg are given by

$$
\left\{\begin{array}{l}
\mathrm{d}_{\mathrm{H}}=\left(2 \ell_{\mathrm{T}} \frac{1-\cos \theta_{\mathrm{T}}}{\theta_{\mathrm{T}}}+\ell_{\mathrm{U}} \sin \theta_{\mathrm{T}}\right) \cos \alpha \\
\mathrm{d}_{\mathrm{V}}=\left(2 \ell_{\mathrm{T}} \frac{1-\cos \theta_{\mathrm{T}}}{\theta_{\mathrm{T}}}+l_{\mathrm{U}} \sin \theta_{\mathrm{T}}\right) \sin \alpha
\end{array}\right.
$$

For all our cases $\theta_{\mathrm{T}}, \theta_{\mathrm{H}}$ and $\theta_{\mathrm{V}}$ are small enough so that these formulas can be approximated as

$$
\left\{\begin{array} { l } 
{ { } ^ { \theta _ { \mathrm { H } } } = \theta _ { \mathrm { T } } \operatorname { c o s } \alpha } \\
{ { } _ { \mathrm { V } } = \theta _ { \mathrm { T } } \operatorname { s i n } \alpha }
\end{array} \quad \left\{\begin{array}{l}
\mathrm{d}_{\mathrm{H}}=\left(\ell_{\mathrm{T}}+\ell_{\mathrm{U}}\right) \theta_{\mathrm{T}} \cos \alpha \\
\mathrm{d}_{\mathrm{V}}=\left(\ell_{\mathrm{T}}+\ell_{\mathrm{U}}\right) \theta_{\mathrm{T}} \sin \alpha
\end{array}\right.\right.
$$

If the same SSC dipoles are used for the MR, only 36 dipoles can be accommodated in a length $8 \ell$. Hence we have the additional condition

$$
\ell_{\mathrm{T}}=\frac{8}{36} \frac{\theta_{\mathrm{T}}}{\theta} l
$$

For a numerical example we use the following values.

1. $\theta_{\mathrm{H}}=24 \theta$. We assume that the projected horizontal bend angle remains the same as in the one-level configuration.

2. $\ell_{U}=11 \ell$. The length of the end utility straights is unchanged so that the matching and the injection configurations are the same as in the one-level case.

3. $\mathrm{d}_{\mathrm{V}}=\ell$. For some of the proposed sites the bedrock is $\sim 400 \mathrm{ft}$ below grade. If one assumes that the Transport section is in the rock (best for tunneling) and the Campus section is near the grade level $\mathrm{d}_{\mathrm{v}}=\ell=114.25 \mathrm{~m}$ is about right. 
From the above equations we have

$$
\mathrm{d}_{\mathrm{V}}=\left(l_{\mathrm{T}}+l_{\mathrm{V}}\right) \theta_{\mathrm{T}} \sin \alpha=\left(\frac{8}{36} \frac{\theta_{\mathrm{T}}}{\theta}+11\right) l_{\theta_{\mathrm{T}}} \sqrt{1-\left(\frac{\theta_{\mathrm{H}}}{\theta_{\mathrm{T}}}\right)^{2}}
$$

or

$$
\frac{\mathrm{d}_{\mathrm{V}}}{l}=\left(11+\frac{8}{36} \frac{\theta_{\mathrm{T}}}{\theta}\right) \sqrt{\theta_{\mathrm{T}}^{2}-\theta_{\mathrm{H}}^{2}}=\theta\left(11+\frac{8}{36} \frac{\theta_{\mathrm{T}}}{\theta}\right) \sqrt{\left(\frac{\theta_{\mathrm{T}}}{\theta}\right)^{2}-24^{2}}
$$

With $\mathrm{d}_{\mathrm{V}} / \ell=1$ this gives the following parameters for the TR:

Bend angle $=\theta_{\mathrm{T}}=39.17 \theta=64.22 \mathrm{mrad}$

Tilt angle $=\alpha=0.9113=52.2^{\circ}$.

Projected horizontal bend angle $=\theta_{\mathrm{H}}=24 \theta=39.35 \mathrm{mrad}$

Projected vertical bend angle $=\theta_{\mathrm{V}}=30.95 \theta=50.75 \mathrm{mrad}$

Length $=\ell_{\mathrm{T}}=8.70 \ell=994 \mathrm{~m}$

and the following parameters for the ring:

Vertical excursion of dogleg $=$ level difference between

Campus and Transport $=\ell=114.25 \mathrm{~m}=375 \mathrm{ft}$

Increment of total tunnel length over the one-level

$$
\text { configuration }=\delta \mathrm{L}=35 l=4.0 \mathrm{~km}
$$

Increment of total bend angle over the one-level

$$
\text { configuration }=\delta \theta=91 \theta=149 \mathrm{mrad}
$$

\section{B) Unipolar Bypass}

Referring to Fig. 5 we see that the outer branch end section ABCDE is topologically the same as the mirrored dogleg of the symmetric bypass denoted as $\mathrm{ABCE}$ in Fig. 4, except in this case the central straight length $l_{U}$ is replaced by $l_{U}+l_{O}$ and we want the projected horizontal bend angle $\theta_{\mathrm{H}}$ of the tilted regions $\widehat{\mathrm{AB}}$ and $\overparen{\mathrm{DE}}$ to be $50 \theta$, the same as that of the one-level configuration. We also keep $\ell_{\mathrm{O}}$ and $l_{\mathrm{I}}$ unchanged so as to get the same separation between the two branches. 
The inner branch end section ABCFG is a little more complicated. The tilted region $\widehat{C F}$ should be a helix with fixed pitch angle $\theta_{\mathrm{V}}$ and

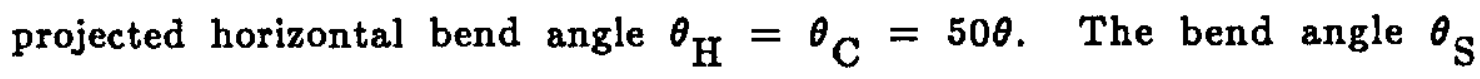
along the helix is given by (see Appendix B)

$$
\sin \theta_{\mathrm{S}}=\sin \theta_{\mathrm{H}} \cos \theta_{\mathrm{V}}
$$

But for values considered here it is a good approximation to simply set

$$
\theta_{\mathrm{S}}=\theta_{\mathrm{H}}=50 \theta
$$

To bend the orbit back to horizontal we need a vertical MR $\left(\ell_{\mathrm{I}}, \theta_{\mathrm{V}}\right)$. It is easy to see that this will bring the 3 inner branch IR's in a horizontal plane roughly on the same elevation as the 3 outer branch IR's. The TR parameters are:

Bend angle $=\theta_{\mathrm{T}}=53.71 \theta=88.07 \mathrm{mrad}$

Tilt angle $=\pi-\alpha=2.7676=158.6^{\circ}$

Projected horizontal bend angle $=\theta_{\mathrm{H}}=50 \theta=81.98 \mathrm{marad}$

Projected vertical bend angle $={ }^{\theta} \mathrm{V}=19.62 \theta=32.18 \mathrm{mrad}$

Length $=l_{\mathrm{T}}=11.93 \mathrm{l}=1363 \mathrm{~m}$

and the parameters of the two-level ring are

Vertical excursion of dogleg $=$ level difference between

Campus and Transport $=l=114.25 \mathrm{~m}=375 \mathrm{ft}$

Increment of total tunnel length over the one-level

$$
\text { configuration }=\delta \mathrm{L}=21.4 \mathrm{l}=2.44 \mathrm{~km}
$$

Increment of total bend angle over the one-level

$$
\text { configuration }=\delta \theta=7.42 \theta=12.2 \mathrm{mad}
$$

Costs, Remarks and Conclusions

Several remarks are in order. 
1. We have not worked out the transport across the long long straight section diametrically opposite the Campus. The design should, however, be straightforward.

2. We have not adjusted the circumferences to give integer if harmonic numbers. But with the very large circumferences this adjustment should be trivially small.

3. In the end $U R$ of the top ring the injection and the switching between branches can both be accommodated in the central $\pm 330 \mathrm{~m}$ long free drift space. One possibility is shown in Fig. 6. The ring orbit is switched between the branches by a switching magnet $W_{D}$ located near the right end of the drift space which deflects the orbit by $\pm 1 / 2$ mrad. The magnet $W_{D}$ is an integral part of the ring and has to ramp up with all the ring magnets.

The injection beam at $1 \mathrm{TeV}$ enters from the left at a downward angle of, say, $3 \mathrm{mrad}+60 \mu \mathrm{rad}$ and is switched to follow the active branch, say branch 1 , by the upstream injection switching magnet $W_{U}$ and the tilted ramped septum $S_{1} \cdot W_{U}$ deflects the beam by $\pm 1 / 2$ mrad in the beam plane and $s_{1}$, deflects the beam horizontally $1 \mathrm{mrad}$ and vertically $3 \mathrm{mrad}$ (total deflection $\sqrt{10} \mathrm{mrad}$ tilted at angle $\tan ^{-1}(1 / 3)$ from vertical). After $S_{1}$ the beam approaches the branch 1 orbit vertically downward at a $60 \mu \mathrm{rad}$ angle and crosses the orbit just beyond $W_{D}$. There it is deflected vertically $60 \mu \mathrm{rad}$ onto the ring orbit by a vertical fast kicker $K_{V}$. The strengths of these magnet elements are given in Fig. 6 and are all quite modest. The dispersions generated by these small deflections although small, should be included in the detailed matching design. 
The end UR of the lower ring has an identical orbit switching system but no injection system.

4) For injection into the two end UR's the most convenient location for the $-1 \mathrm{~km}$ radius High Energy Booster (HEB) is in the middle of the Campus straddling the two branch lines as shown in Fig. 7. The major advantage of this arrangement is that bipolar operation of the HEB is not needed. Beams extracted from diametrically opposite straight sections of HEB are channeled by long but relatively straight transport lines to the end UR's. These transport lines and the tunnels housing them could be quite simple and inexpensive.

If the HEB is capable of bipolar operation one can extract oppositely circulating beams from the same straight section and inject them into neighboring utility straights of the SSC rings as in Case A of Ref. 2. This arrangement, in addition to requiring the hard bipolar operation of a superconducting HEB, would need a longer Campus.

5) With the injection geometry shown in Fig. 7, for all 4 configurations-symmetric and unipolar bypasses, one- and two-levels--the minimum size of the Campus to enclose the injector and all 6 IR's is about $5 \mathrm{~km}$ long by $2.5 \mathrm{~km}$ wide. To this one must add the abort/external beams area, the arc areas, the service and access areas, and the necessary easements.

6) To make some rough estimates of the cost differentials we shall use the 1987 unit-costs given by the Central Design Group, namely

Tunnel cost $\left(\mathrm{C}_{\mathrm{T}}\right): \quad \$ 4 \mathrm{M} / \mathrm{km}$

Magnet cost $\left(C_{M}\right)$ : $\quad \$ 0.12 \mathrm{M} /$ dipole (including distributed quadrupole cost)

$$
\mathrm{EDIA}=15 \%\left(\mathrm{C}_{\mathrm{T}}+\mathrm{C}_{\mathrm{M}}\right)
$$




$$
\text { Contingency }=25 \%\left(\mathrm{C}_{\mathrm{T}}+\mathrm{C}_{\mathrm{M}}+\mathrm{EDIA}\right)
$$

The incremental costs for the one-campus and the two-level configurations are given in the table below (two rings).

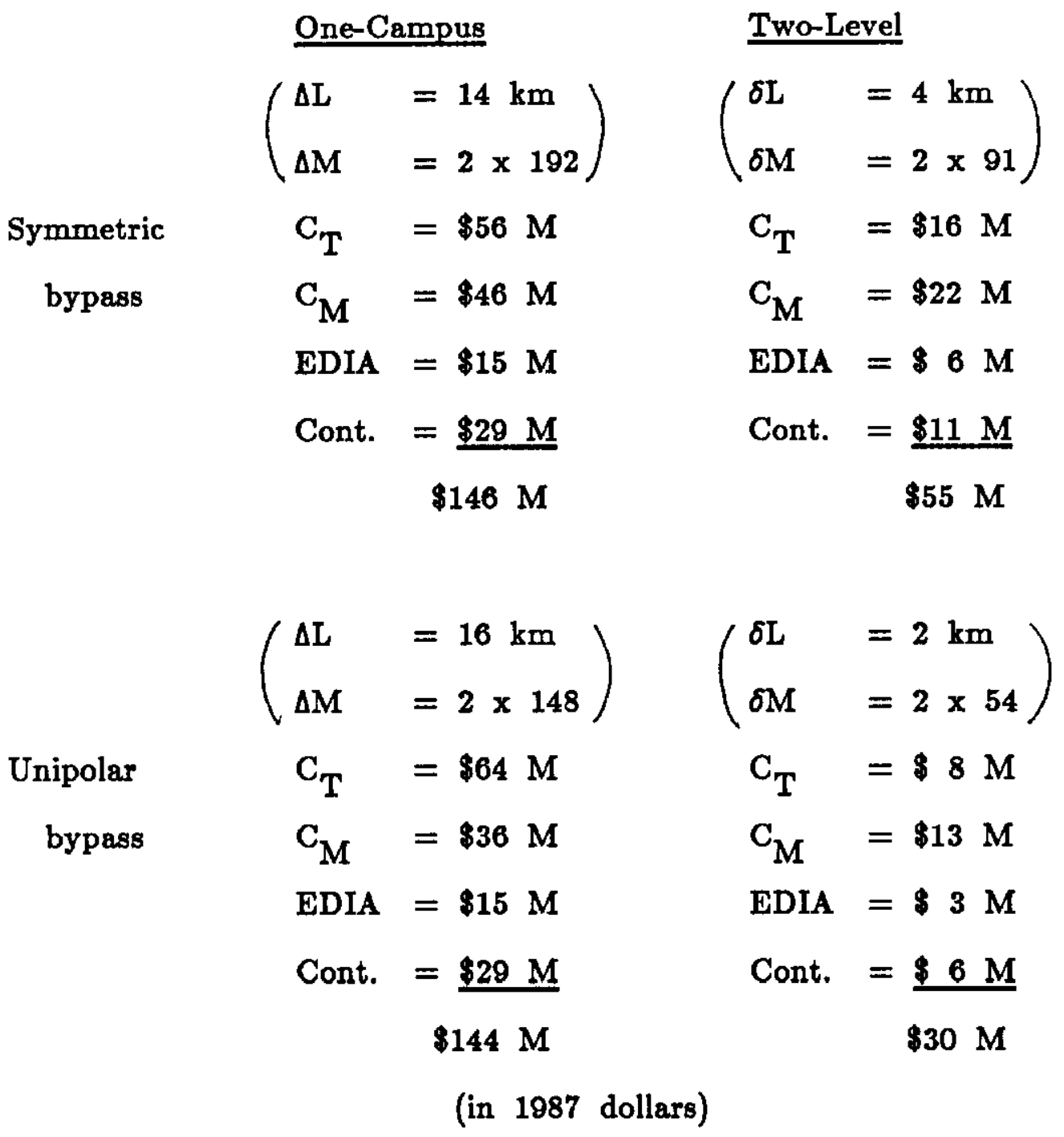

This table shows that the incremental cost of the Unipolar Bypass configuration is slightly less. On the other hand, the Symmetric Bypass configuration is simpler and neater and has many construction, alignment and operation advantages which are well worth the slightly higher cost. 
Appendix A - RMS cone angle and radius of the muon beam from an IP.

The high energy muons from an IP are produced essentially at $0^{\circ}$ (or $180^{\circ}$ ). When they go through a thickness $\ell_{\mu}$ of earth shielding all muons with parallel momentum

$$
\mathrm{p}_{\|}<\left(0.4 \frac{\mathrm{TeV}}{\mathrm{c}} / \mathrm{km}\right) \ell_{\mu}
$$

are ranged out. Here we have taken

$$
\left\{\begin{array}{l}
\frac{d \mathrm{E}}{\mathrm{dx}}-2 \mathrm{YeV} / \mathrm{g} / \mathrm{cm}^{2} \text { and } \\
\text { Density of earth }-2 \mathrm{~g} / \mathrm{cm}^{3}
\end{array}\right.
$$

The muons that penetrated the earth shielding will have gained an rms perpendicular momentum through multiple Coulomb scattering of the amount

$$
\mathbf{p}_{\perp}=(1.5 \mathrm{GeV} / \mathrm{c}) \sqrt{\ell_{\mu}(\mathrm{km})}
$$

Where we have taken the radiation length of earth to be $25 \mathrm{~g} / \mathrm{cm}^{2}$. Thus, the rms cone angle of the muon beam is

$$
\theta_{\mu} \equiv \frac{p_{\perp}}{p_{\mid l}}=\frac{4 \mathrm{mrad}}{\sqrt{l_{\mu}(\mathrm{km})}}
$$

and the rms radius of the $\mu$-beam after going through $\ell_{\mu}$ length of earth is

$$
\sigma_{\mu}=\theta_{\mu} \ell_{\mu}=(4 \mathrm{~m}) \sqrt{\ell_{\mu}(\mathrm{km})}
$$

All IP's should be located several $\sigma_{\mu}$ away from one another's muon beams. 
Appendix B - Geometry of tilted dogleg orbit and helix orbit

1) One half of the dogleg is shown in Fig. B1. The orbit is the curve $\mathrm{ABC}, \mathrm{A}$ being the start and $\mathrm{C}$ being the midpoint of the dogleg. The angles involved are

Tilt angle $=\alpha=\angle A O D$

Bend angle $=\theta_{\mathrm{T}}=\angle \mathrm{AOB}=\angle \mathrm{BPQ}$

Projected horizontal bend angle $=\theta_{\mathrm{H}}=\angle \mathrm{EPQ}$

Projected vertical bend angle $=\theta_{\mathrm{V}}=\angle \mathrm{GPQ}$

The relationship between the angles are

$$
\left\{\begin{array}{l}
\tan \theta_{\mathrm{H}}=\frac{\mathrm{EQ}}{\mathrm{PQ}}=\frac{\overline{\mathrm{BQ}}}{\mathrm{PQ}} \cos \alpha=\tan \theta_{\mathrm{T}} \cos \alpha \\
\tan \theta_{\mathrm{V}}=\frac{\mathrm{GQ}}{\mathrm{PQ}}=\frac{\mathrm{BQ}}{\mathrm{PQ}} \sin \alpha=\tan \theta_{\mathrm{T}} \sin \alpha
\end{array}\right.
$$

The lateral excursions are

$$
\left\{\begin{aligned}
\mathrm{d}_{\mathrm{H}}=2 \overline{\mathrm{DU}} & =2 \overline{\mathrm{AW}} \cos \alpha=2\left(\widehat{\mathrm{AB}} \frac{1-\cos \theta_{\mathrm{T}}}{\theta_{\mathrm{T}}}+\overline{\mathrm{BC}} \sin \theta_{\mathrm{T}}\right) \cos \alpha \\
& =2\left(\ell_{\mathrm{T}} \frac{1-\cos \theta_{\mathrm{T}}}{\theta_{\mathrm{T}}}+\frac{\ell_{\mathrm{U}}}{2} \sin \theta_{\mathrm{T}}\right) \cos \alpha \\
\mathrm{d}_{\mathrm{V}}=2 \overline{\mathrm{FV}} & =2 \overline{\mathrm{AW}} \sin \alpha \\
& =2\left(l_{\mathrm{T}} \frac{1-\cos \theta_{\mathrm{T}}}{\theta_{\mathrm{T}}}+\frac{l_{\mathrm{U}}}{2} \sin \theta_{\mathrm{T}}\right) \sin \alpha
\end{aligned}\right.
$$

2) A section of a helix orbit is shown in Fig. B2 as $\overparen{A E}$. Plane ABCD is tangent to the orbit cylinder and $\overline{\mathrm{AC}}$ is tangent to the orbit at $\mathrm{A}$. Similarly EFGH is the tangent plane and $\overline{\mathrm{EG}}$ is tangent to the orbit at E. We have also made $\overrightarrow{\mathrm{AC}}=\overline{\mathrm{EG}}$. Plane $\mathrm{ABCD}$ is parallel-translated to EIKH. The angles are

Orbit bend angle from $A$ to $E=\theta_{S}=\angle K E G$

Horizontal projection of bend angle $=\theta_{\mathrm{H}}=\angle \mathrm{IEF}$

Vertical pitch angle of helix $=\theta_{\mathrm{V}}=\angle \mathrm{KEI}=\angle \mathrm{GEF}$ 
The relation between these angles is

$$
\frac{\sin \theta_{\mathrm{S}}}{\sin \theta_{\mathrm{H}}}=\frac{\mathrm{GR} / \mathrm{ER}}{\mathrm{FI} / \mathrm{EI}}=\frac{\mathrm{EI}}{\mathrm{EK}}=\cos \theta_{\mathrm{V}}
$$

References

1. L. M. Lederman "A One Lab SSC Configuration" Proc. of the 1986 Summer Study on the Physics of the SSC, p. 518

2. L. M. Lederman and L. C. Teng "A One-Campus SSC" Fermilab report TM-1452 (April 1987)

3. A. A. Garren and D. E. Johnson "The $90^{\circ}$ (September 1987) SSC Lattice" SSC-146. 


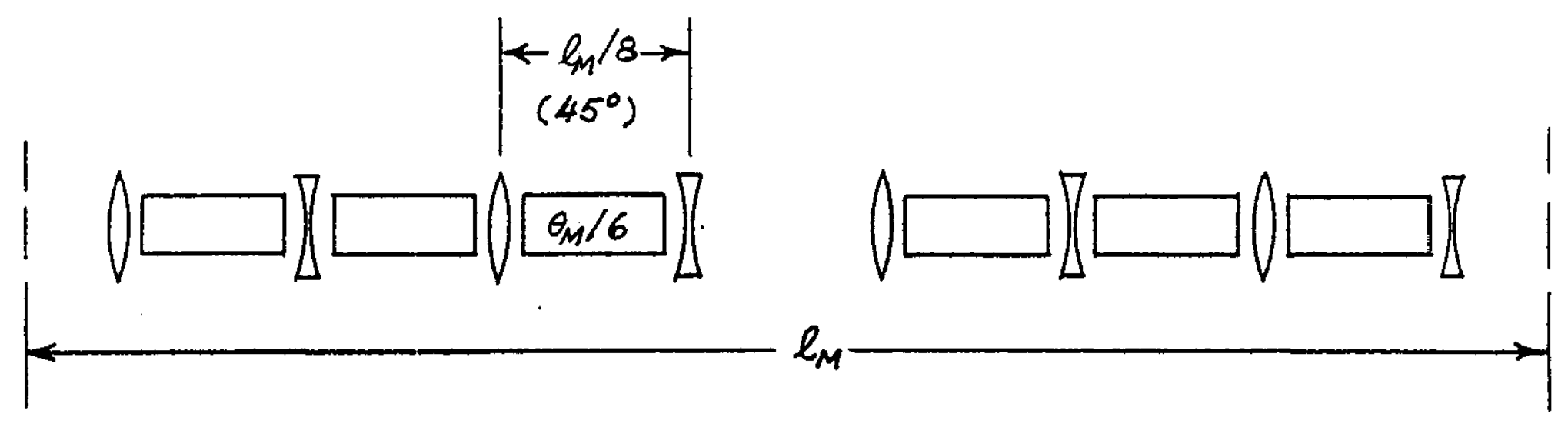

Figure 1. An all purpose matching region denoted as $\operatorname{MR}\left(\ell_{\mathrm{M}}, \theta_{\mathrm{M}}\right)$ which matches zero dispersion to zero dispersion and has unity transfer matrices in both transverse planes and adjustable length $\ell_{M}$ and bend angle $\theta_{\mathrm{M}}$ 


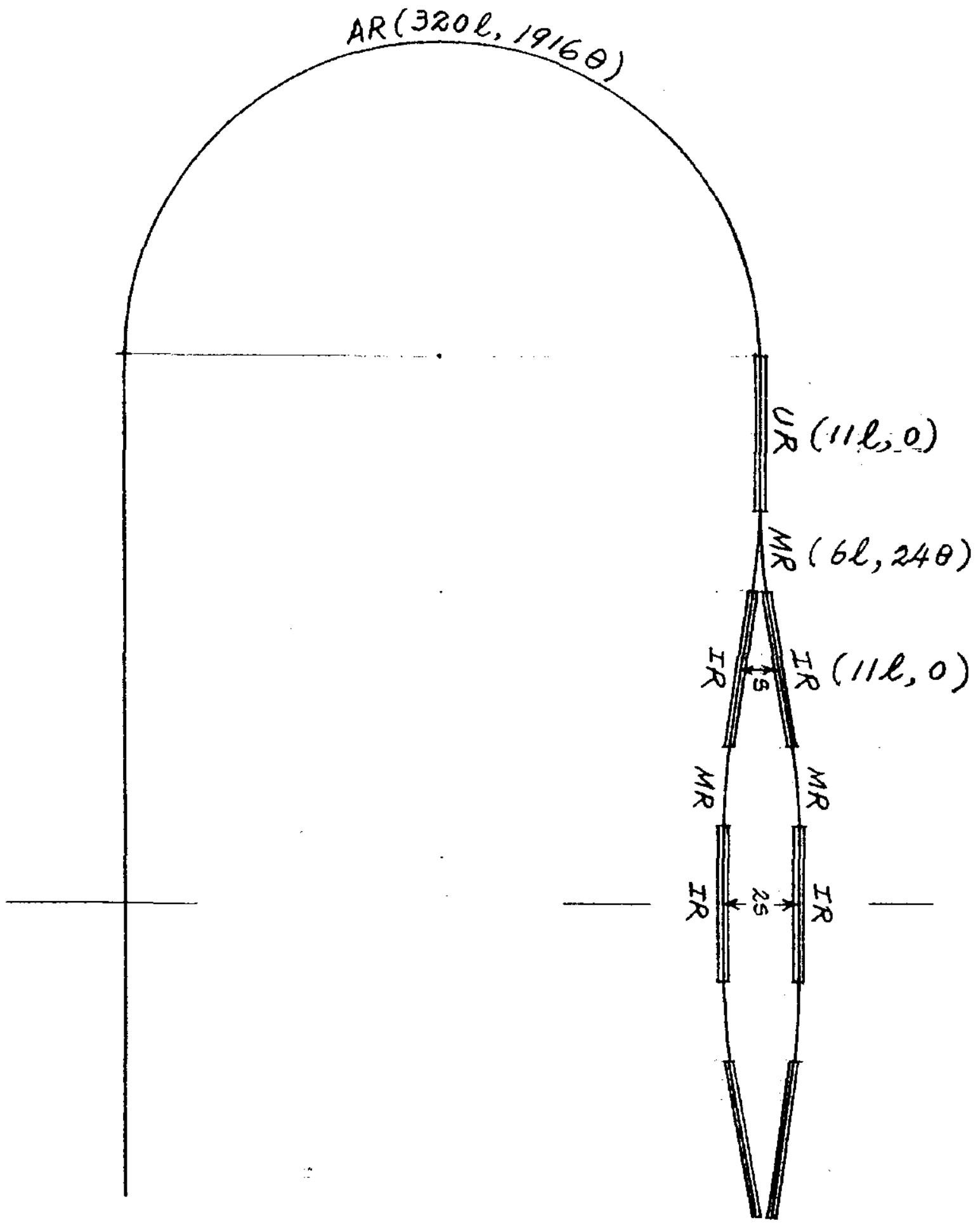

Figure 2. One-campus one-level configuration with symmetric bypass (not to scale). 


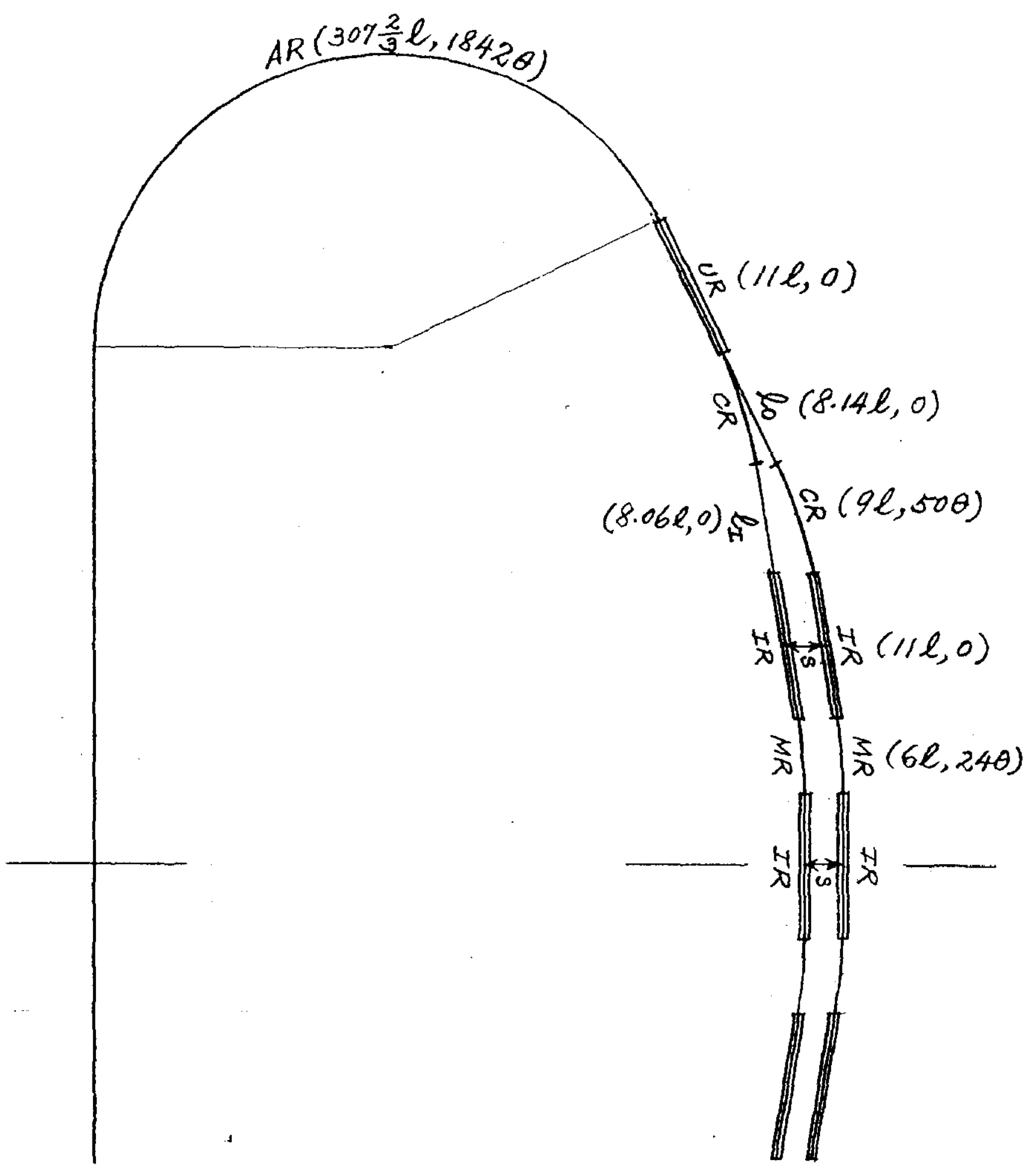

Figure 3. One-campus one-level configuration with unipolar bypass (not to scale). 


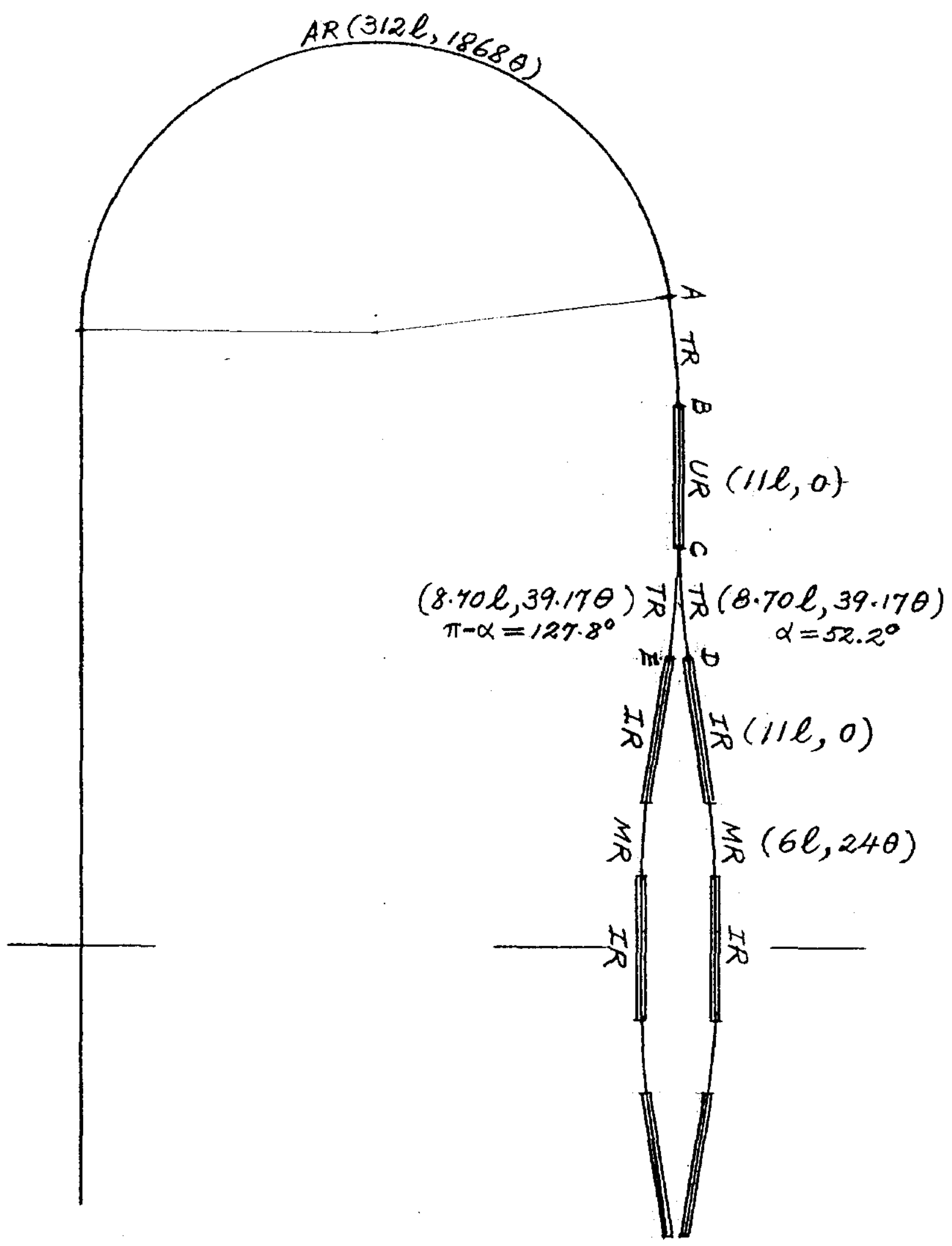

Figure 4. Two-level configuration with symmetric bypass (not to scale). 


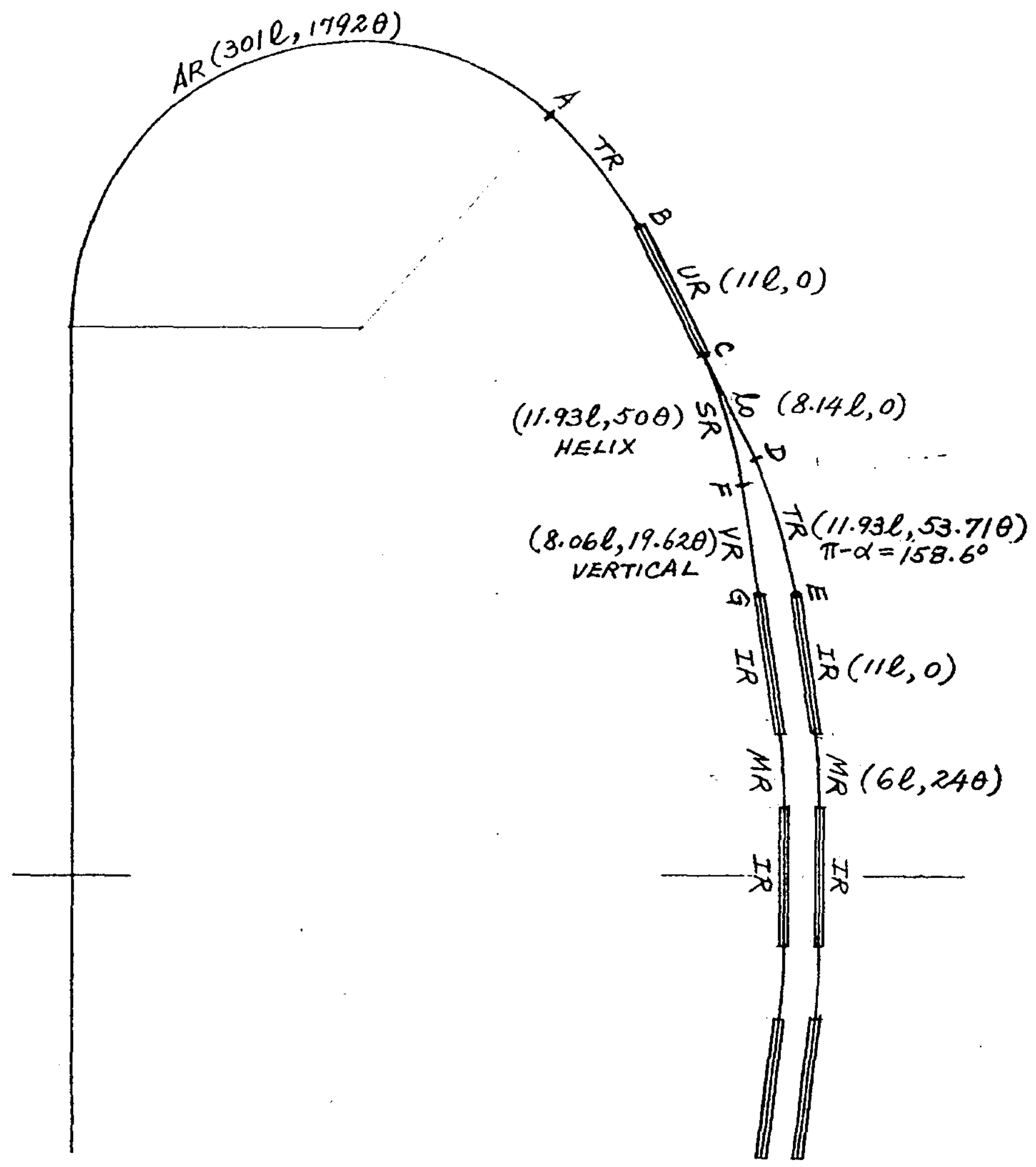

Figure 5. Two-level configuration with unipolar bypass (not to scale). 


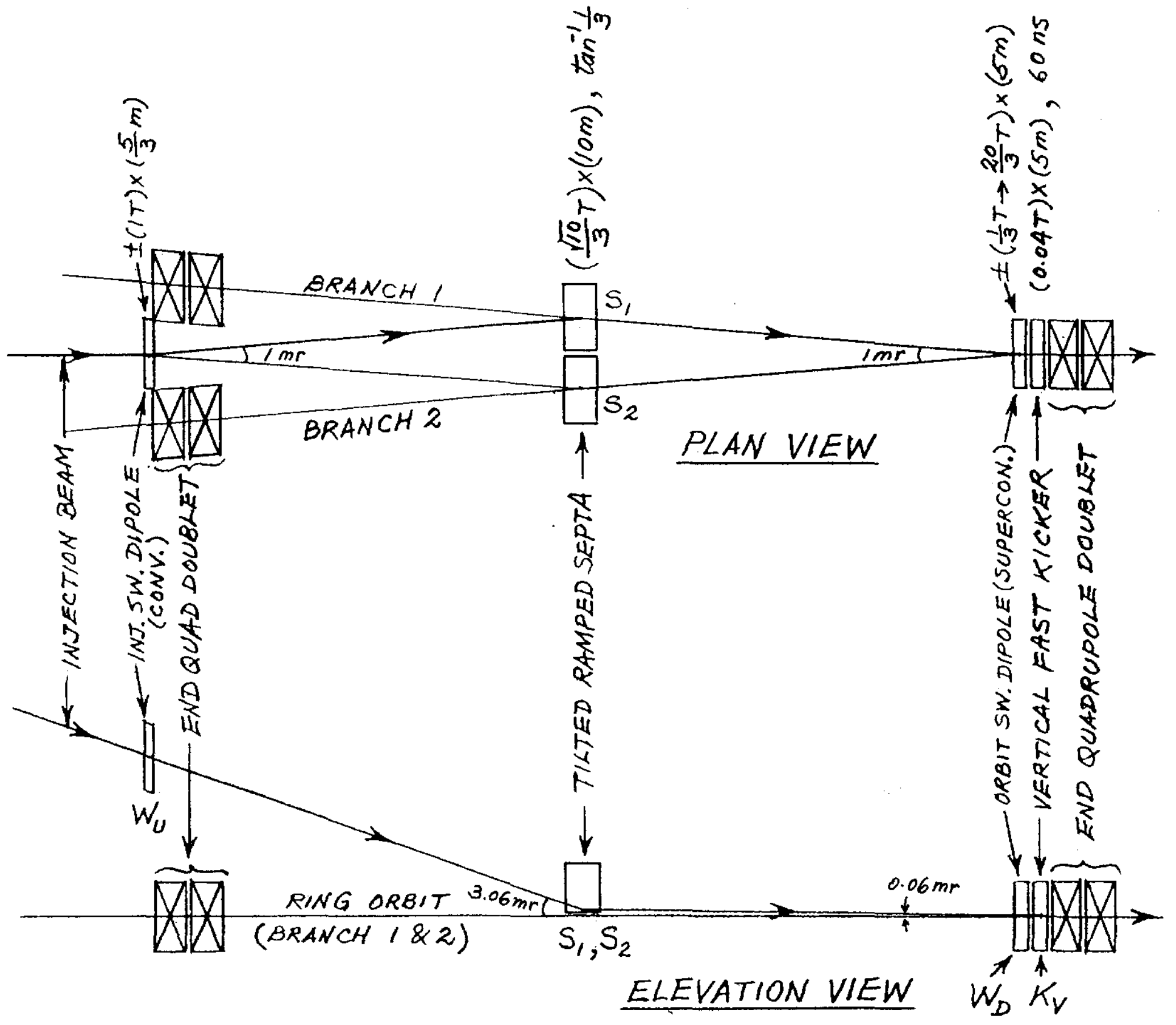

Figure 6 . Injection and branch-switching systems in the central drift space of an end utility region (not to Bcale). 


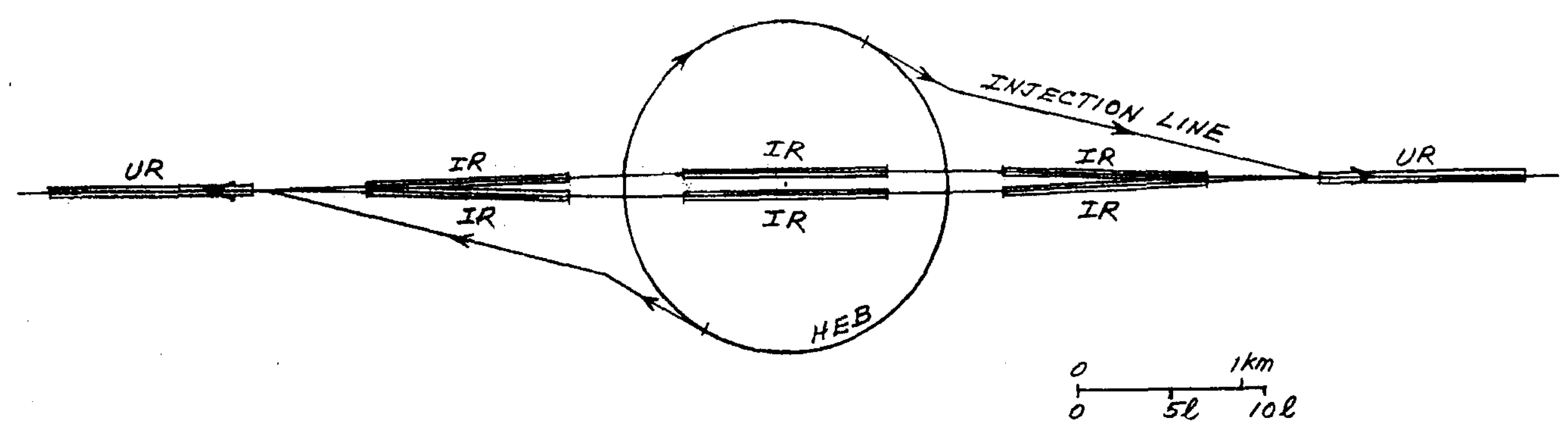

Figure 7. Optimal positioning of a unipolar high energy booster injector and the injection transport lines. 


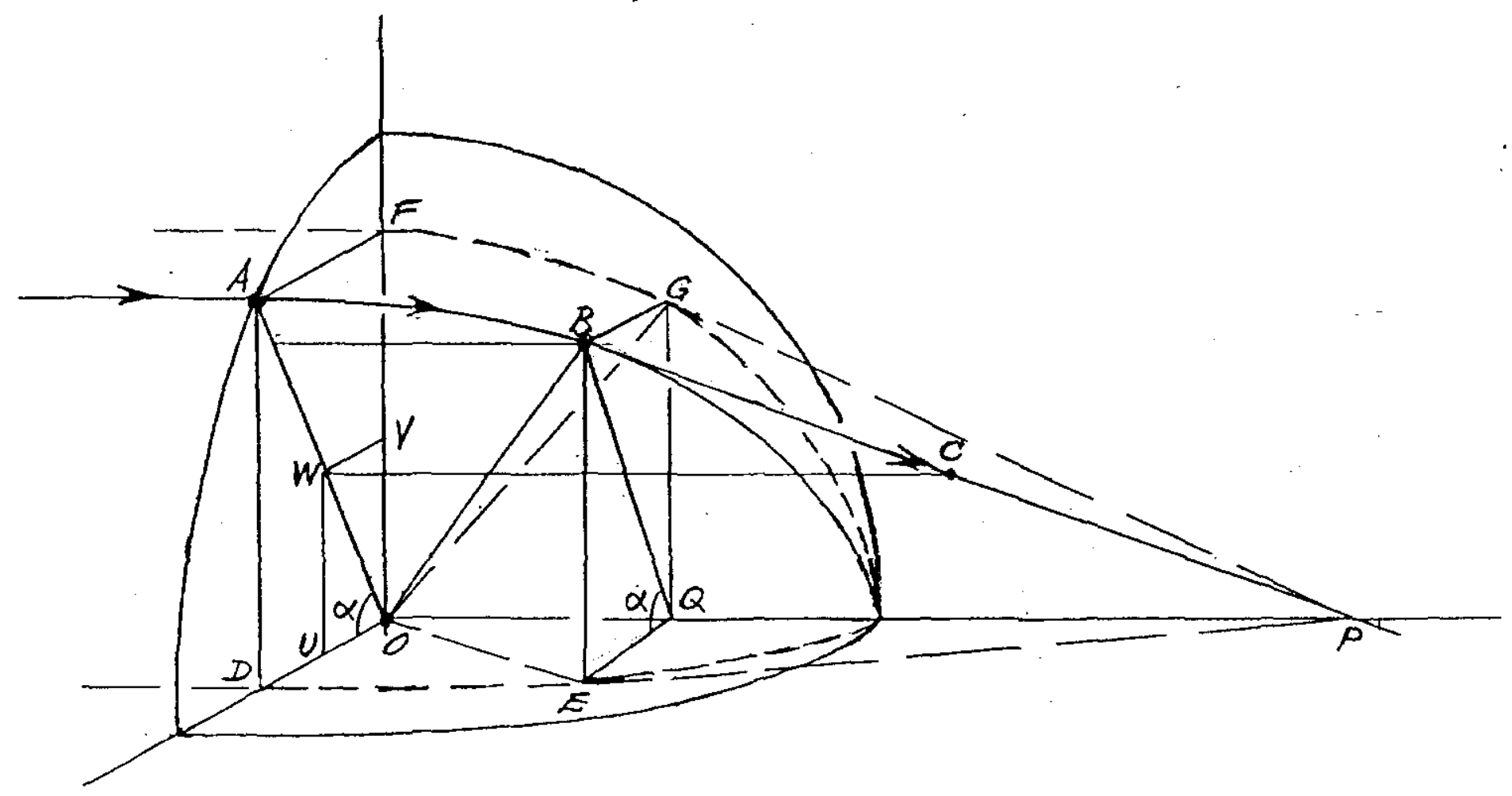

Figure B1. Geometry of a tilted dogleg orbit. 


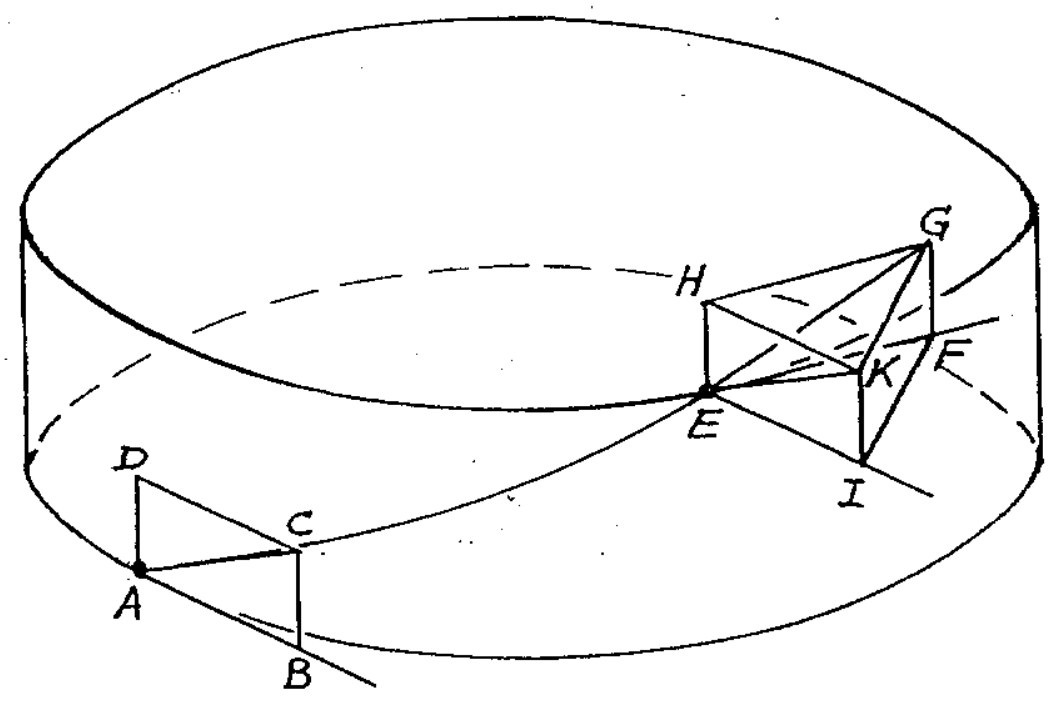

Figure B2. Geometry of a helix orbit. 